\title{
A fetal scalp electrode as a simple aid in the search for a lost needle fragment during sacrospinous ligament fixation
}

\author{
Marieke P. G. Schoonenberg • Kirsten B. Kluivers • \\ Mark E. Vierhout
}

Received: 30 April 2010 /Accepted: 1 August 2010 / Published online: 27 August 2010

(C) The Author(s) 2010. This article is published with open access at Springerlink.com

\begin{abstract}
A needle fragment was lost during a sacrospinous ligament fixation. This was recognized during the procedure, but could not be found at that moment. The patient complained of severe buttock pain postoperatively. The needle fragment was localized on CT scan of the pelvis. A fetal scalp electrode helped as a search device to localize the needle on X-ray during the secondary surgery. The patient was operated successfully and was free of pain after 6 weeks.
\end{abstract}

Keywords Buttock pain · Fetal scalp electrode $\cdot$ Needle . Sacrospinous ligament fixation

\section{Introduction}

Vaginal vault prolapse is known as a late complication of hysterectomy, and the incidences reported range between $0.2 \%$ and $43 \%$ [1]. Sacrospinous ligament fixation (SSLF) is a frequently used procedure for the treatment of patients with vaginal vault prolapse. Several studies have shown that it is a safe and effective procedure, although postoperative pain may occur. Other complications described are hemorrhage, cystitis, perforation of the bladder, rectum or small bowel, rectovaginal fistula, and nerve injury. Nerve injuries specific to SSLF may result in buttock, anal, and vulvar pain $[1,2]$. Despite the well-recognized difficult access of the sacrospinous ligament (SSL), loss of a needle fragment has never been described during this procedure.

M. P. G. Schoonenberg $(\triangle) \cdot$ K. B. Kluivers $\cdot$ M. E. Vierhout Radboud University Nijmegen Medical Centre, Internal postal code 791 P.O. Box 9101, 6500 HB Nijmegen, The Netherlands

e-mail: m.schoonenberg@obgyn.umcn.nl

URL: www.umcn.nl

\section{Case}

A 48-year-old woman free from comorbidity, para 2, was referred to our hospital with pain in her right buttock after loss of a needle fragment during SSLF for a vaginal vault prolapse.

She had undergone a right-sided unilateral SSLF in combination with posterior colporrhaphy for second degree vaginal vault prolapse and third degree rectocele (according to Baden Walker classification). During this procedure, two nonresorbable multifilament sutures were placed under vision through the right SSL. Unfortunately, the needle broke on placement of the last suture, and approximately $3 / 4$ of the needle was lost. The needle fragment was not palpable or visible. X-ray was used to localize the needle. The further search was considered to embear the risk of more complications such as nerve injuries. Thus, the search was abandoned, and it was left in the patient. There were no other intraoperative complications.

After the operation, the patient was discharged without any complaints. Information on the complication of the lost needle fragment was provided, including the considerations as why not to remove the needle fragment, as well as the expectation that the lost needle fragment would stay asymptomatic and undangerous. However, 2 weeks later, the patient presented with pain in her right buttock, mainly during exercise. The patient had a normal temperature and infectious parameters in the blood. The abdomen was soft and non-tender, and vaginal examinations revealed no abnormalities. Computed tomography (CT) scan of the pelvis was performed and showed the $17-\mathrm{mm}$ needle fragment near the sacrospinous-coccygeal ligament complex on the right side (Fig. 1).

At first, it was decided to wait and hope for spontaneous recovery instead of undertaking immediate secondary surgery. Three months later, there was no change in pain, 


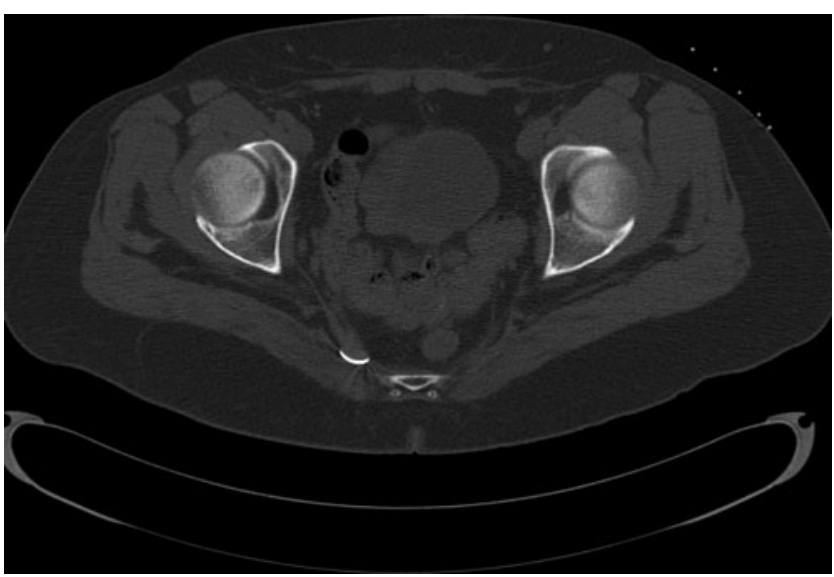

Fig. 1 Transversal CT image demonstrates the 17-mm needle fragment near the sacrospinous-coccygeal ligament complex on the right side

and the patient was referred to our tertiary urogynecological center for a second surgery. A midline incision in the posterior vaginal wall was performed, and the right side of the rectum was separated from the posterior vaginal wall. A right-sided mediolateral episiotomy was placed for better access. Through sharp and blunt dissection, the right sacrospinous ligament was made visible. The needle fragment was not perceptible by palpation. An orthopedic surgeon, experienced in $3 \mathrm{D}$ spatial comprehension of $2 \mathrm{D}$ $\mathrm{X}$-ray images, assisted in the procedure. A fetal scalp electrode was placed on the sacrospinous-coccygeal ligament. On X-ray, the needle fragment was visible in relation to the fetal scalp electrode (Fig. 2), resulting in a safe removal of the detected needle.

The day after the operation, the patient could be discharged home. On follow-up 6 weeks later, the patient was free of symptoms of pain, fever, vaginal discharge, or recurrent prolapse.

\section{Discussion}

This report describes the first case of a lost needle fragment as the cause of buttock pain after SSLF. Furthermore, there are no previous reports on a fetal scalp electrode as a simple aid in the search for a needle fragment.

The prevalence of postoperative buttock pain after SSLF is estimated at $10-15 \%$. The pain can be explained by injury to surrounding nerves of the sacral plexus and branches of the pudendal nerve. Buttock pain will resolve spontaneously in most cases [2]. In this case, buttock pain was thought to be caused by the lost needle fragment, and indeed, the pain was relieved after removal.

As has been reported previously, needle fragments mostly do not cause pain [4]. Nonetheless, all efforts to remove the fragment during initial surgery should be undertaken because the fragment can migrate due to the pointed shape on one end. This makes movement in solid and moving tissue possible, such as muscle. In the present case, the pain might be explained by the sole presence of the needle fragment itself, or due to migration into moving tissue and secondary irritation of the nerves that run through the sacrospinouscoccygeal ligament complex. There can also be a strong psychoemotional component in these cases, and sometimes, only reassurance may be needed.

The complication of a lost needle fragment has not been described in major urogynaecological textbooks. One previous case report in association with uterovaginal prolapse treatment could be found in the literature. Malhotra has described a patient with chronic pain since a Fothergills repair 9 years previously. During extensive investigations, a needle was incidentally detected. Removal of the needle relieved the patient of her symptoms [4].

There are a few further reports on a lost needle during other vaginal procedures [3-5]. The incidence of a needle fracture during episiotomy repair has been reported to be $0.17 \%$ [3]. Hösli et al. have presented a case of a lost broken needle after suturing a vaginal tear following delivery [3].

$\mathrm{X}$-ray examination during surgery has been recommended when searching for a lost needle fragment. During surgery, a magnet might help as a rapid, cheap, and safe method. Until now, however, there are no sterile-packed magnets that could be used for the purpose [5].

In this case, we used a fetal scalp electrode as a search device. The right SSL was made visible by sharp and blunt dissection. The fetal scalp electrode was placed on the SSL. The interpretation of the transversal and lateral 3-D CT images in combination with the 2D X-ray images of the needle and the fetal scalp electrode during surgery made it

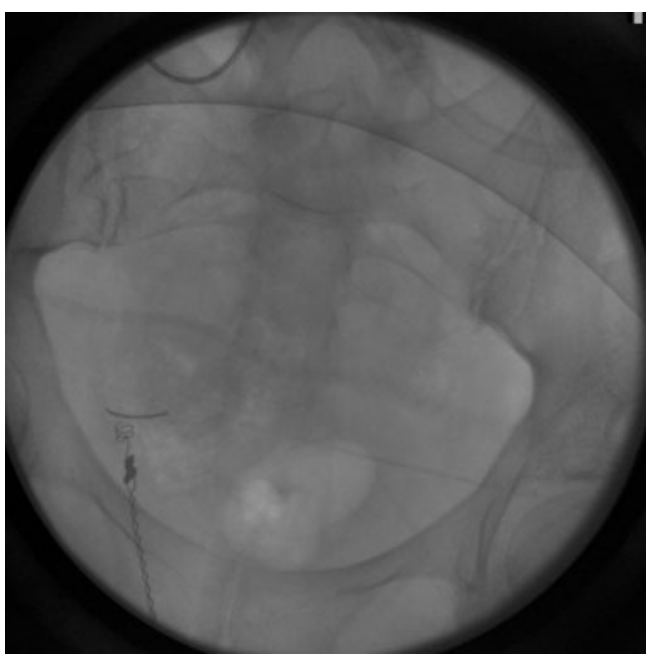

Fig. 2 X-ray demonstrates the 17-mm needle fragment 
possible to imagine the depth and the exact location of the needle, resulting in a safe removal of the detected needle.

In summary, we describe a case of a lost needle fragment during sacrospinous fixation which could be removed 4 months after the initial procedure. Helpful elements were the use of a right-sided episiotomy and the use of a fetal scalp electrode as a search device.

\section{Conflicts of interest None.}

Open Access This article is distributed under the terms of the Creative Commons Attribution Noncommercial License which permits any noncommercial use, distribution, and reproduction in any medium, provided the original author(s) and source are credited.

\section{References}

1. Sze EH, Karram MM (1997) Transvaginal repair of vault prolapse: a review. Obstet Gynecol 89:466-475

2. Dietz V, Huisman M, de Jong J, Heintz P, van der Vaart C (2008) Functional outcome after sacrospinous hysteropexy for uterine descensus. Int Urogynecol J 19:747-752

3. Hösli I, Tercanli S, Holzgreve W (2000) Complications of lost needle after suture of vaginal tear following delivery. Arch Gynecol Obstet 264:159-161

4. Malhotra N, Roy K, Sinha A (2003) Forgotten needle - an unusual cause of chronic pelvic pain. Arch Gynecol Obstet 269:68-69

5. Möhrenschlager M, Ring J, Henkel V, Jessberger B (2008) Lost needle: a simple search device for the operating room's floor. Langenbecks Arch Surg 393:1009-1011 